01.3

\title{
Мультистабильность и сложные колебательные режимы в генераторе с запаздывающим отражением от нагрузки
}

\author{
(С) М.И. Балакин ${ }^{1}$, Н.М. Рыскин ${ }^{2,3}$ \\ ${ }^{1}$ Саратовский государственный технический университет им. Гагарина Ю.А, Саратов, Россия \\ ${ }^{2}$ Саратовский филиал Института радиотехники и электроники им. В.А. Котельникова РАН, Саратов, Россия \\ ${ }^{3}$ Саратовский национальный исследовательский государственный университет им. Н.Г. Чернышевского \\ E-mail: balakinmaxim@gmail.com
}

Поступило в Редакцию 8 октября 2018г.

В окончательной редакции 8 октября 2018г.

Принято к публикации 25 декабря 2018г.

\begin{abstract}
Проведено исследование особенностей формирования мультистабильности в генераторе с запаздывающим отражением от нагрузки. Определены характерные сценарии рождения и эволюции мультистабильных состояний. Выявлено влияние неизохронности на сценарии перехода к хаосу.
\end{abstract}

DOI: 10.21883/PJTF.2019.06.47497.17551

Нелинейная динамика систем с запаздыванием в настоящее время является предметом активных исследований, поскольку подобные системы играют большую роль во многих областях науки и техники: радиофизике, механике, кибернетике, нелинейной оптике, биологии, медицине, экономике (см., например, [1,2]). В частности, в последнее время привлекла внимание задача о воздействии запаздывающего сигнала, отраженного от удаленной нагрузки, на динамику различных микроволновых электронных генераторов, в том числе гиротронов [3-5]. Как известно, наличие запаздывания приводит к появлению нескольких устойчивых стационарных состояний, т.е. к мультистабильности и гистерезису [6]. Эффекты мультистабильности могут носить паразитный характер, приводя к скачкообразным переключениям между режимами генерации на различных модах при флуктуациях управляющих параметров. С другой стороны, наличие мультистабильности можно использовать, например, для кодирования и передачи информации [7]. Отражения также могут приводить к появлению автомодуляционных и хаотических режимов генерации [8].

Типичным примером автоколебательной системы с запаздыванием является генератор ван дер Поля-Дуффинга с отражением от удаленной нагрузки

$$
\frac{d^{2} x}{d t^{2}}-\left(\alpha-x^{2}\right) \frac{d x}{d t}+\omega_{0}^{2} x+\gamma x^{3}=\omega_{0}^{2} k x(t-\tau)
$$

который может служить простейшей моделью доплеровского автодина [9]. В уравнении (1) $x$ - динамическая переменная, $\alpha$ - управляющий параметр, характеризующий превышение порога генерации, $\omega_{0}-$ собственная частота малых колебаний, $\gamma$ - параметр неизохронности, $k-$ коэффициент отражения, $\tau-$ время запаздывания. В [9] показано, что при увеличении параметров $k$ и $\tau$ режим периодических автоколебаний теряет устойчивость и возникают квазипериодические колебания, а затем каскад бифуркаций удвоения тора, завершающийся возникновением хаоса. Однако представляется, что наличие мультистабильности должно существенно повлиять на картину сложных колебательных режимов. В настоящей работе выявлены бифуркационные механизмы формирования мультистабильности в генераторе с запаздывающим отражением, что важно с точки зрения управления переходами между сосуществующими колебательными модами и подавления нежелательных колебаний.


Рис. 1. $a-$ бифуркационная диаграмма на плоскости параметров $\tau-k ; b-$ общая структура зон генерации: $O-$ область устойчивости состояния равновесия в начале координат, 2-3 и 3-4 - перекрытие зон генерации различных мод (области мультистабильности). 


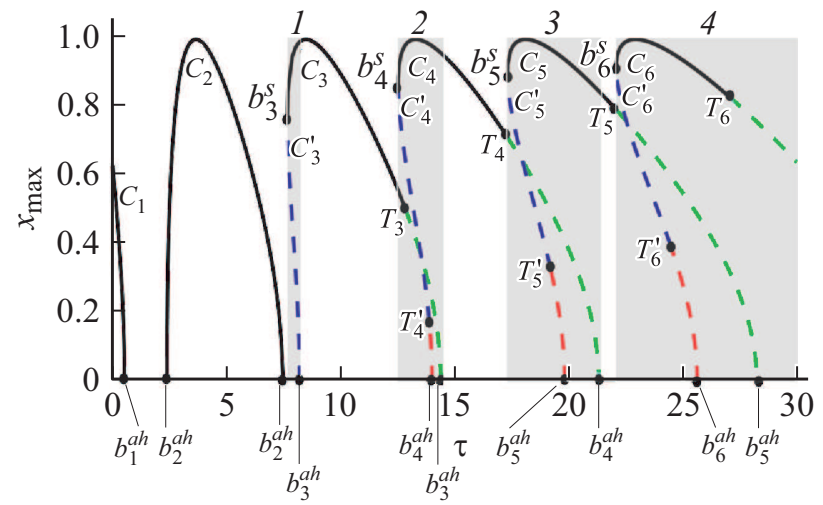

Рис. 2. Однопараметрическая бифуркационная диаграмма в зависимости от запаздывания $\tau$. $k=0.2$.

На рис. 1, $a$ приведена структура плоскости параметров $\tau-k$, построенная с помощью пакета бифуркационного анализа DDE-BIFTOOL [10]. Значения остальных параметров следующие: $\alpha=0.1, \omega_{0}=1.0, \gamma=1.0$. При этом в генераторе без отражений реализуется режим квазигармонических автоколебаний. В целом всю диаграмму можно разделить на отдельные листы, соответствующие режимам генерации различных собственных мод (рис. $1, b$ ). Границы этих листов формируются отрезками линий бифуркации Андронова-Хопфа $A H_{n}$ и касательных бифуркаций $S_{n}$ (здесь $n=1,2, \ldots$, моды пронумерованы в соответствии с рис. $1, b$ ). При пересечении линий $A H_{n}$ происходит рождение предельного цикла из состояния равновесия в начале координат, а при пересечении линий $S_{n}$ - рождение устойчивого и неустойчивого предельных циклов из сгущения фазовых траекторий. Таким образом, если увеличивать коэффициент отражения, при пересечении какой-либо из данных линий происходит возбуждение периодических колебаний на соответствующей собственной моде. В области перекрытия листов, соответствующих различным модам, наблюдаются мультистабильность и формирование сложных колебательных режимов. Области устойчивости неподвижной точки обозначены символом $O$.

Таким образом, внутри каждого из листов существует устойчивый предельный цикл, претерпевающий различные бифуркации при вариации управляющих параметров. При увеличении коэффициента отражения он теряет устойчивость и наблюдается возбуждение квазипериодических колебаний в результате суперкритической бифуркации Неймарка-Сакера (штриховые линии $N_{n}$ ). Штрихпунктирными линиями $N_{n}^{\prime}$ отмечены субкритические бифуркации Неймарка-Сакера неустойчивых циклов.

Более подробно эту картину иллюстрирует представленная на рис. 2 бифуркационная диаграмма, на которой отложены максимумы переменной $x$ в зависимости от $\tau$ при $k=0.2$ (вдоль горизонтальной пунктирной линии на рис. $1, a$ ). Сплошными линиями показаны устойчивые режимы, штриховыми - неустойчивые. Точки $b_{n}^{s}$ соответствуют пересечениям линий касательной бифуркации $S_{n}$. Видно, что при этом рождается пара предельных циклов: устойчивый $C_{n}$ и неустойчивый $C_{n}^{\prime}$. Точки $b_{n}^{a h}$ отвечают пересечениям линий суб- или суперкритической бифуркации Андронова-Хопфа $A H_{n}$, при этом происходит рождение циклов из неподвижной точки. Точки $T_{n}\left(T_{n}^{\prime}\right)$ соответствуют бифуркациям рождения устойчивого (неустойчивого) тора из устойчивого (неустойчивого) цикла. Мультистабильность имеет место в областях, отмеченных серым на рис. 2, причем наблюдается сосуществование устойчивой неподвижной точки и предельного цикла (область 1 ), двух устойчивых предельных циклов (область 2), устойчивого предельного цикла и устойчивого тора (области 2-4), двух устойчивых торов (область 4). Таким образом, обнаруженная картина поведения оказывается значительно более сложной, чем представлялось ранее [9].

При увеличении коэффициента отражения будет наблюдаться мультистабильность квазипериодических и хаотических режимов, соответствующих различным собственным модам. Более того, как показало численное моделирование, переходы к хаосу могут происходить по различным сценариям. Наряду с описанным в [9] переходом через последовательность удвоений торов наблюдается сценарий разрушения квазипериодического движения Рюэля-Такенса. То, какой именно из двух сценариев реализуется, зависит от параметра неизохронности $\gamma$. На рис. 3 построены границы рождения квазипериодических колебаний и перехода к хаосу на плоскости параметров $\gamma-k$ при $\tau=20$. Удвоения двухчастотных торов наблюдаются только при достаточно больших $\gamma$ (кружки на рис. 3), тогда как при меньших $\gamma$ происходит рождение трехчастотного тора и его последующее разрушение (квадраты на рис. 3).

Отметим, что пороговые значения растут при уменьшении неизохронности. Более того, при $\gamma<1.2$ граница перехода к хаосу сдвигается в область $k>1$, рассматривать которую не имеет смысла, поскольку $k$ представляет собой коэффициент отражения. Это может быть причиной того, что в работах [4-6] хаотические режимы не наблюдались.

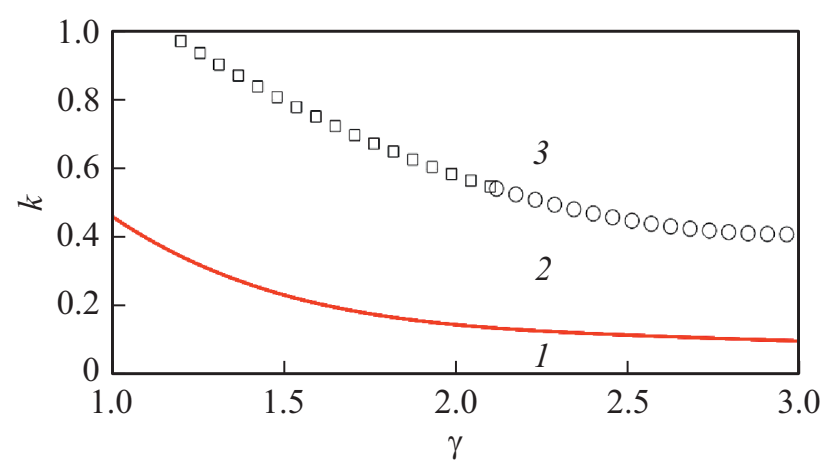

Рис. 3. Характерные области генерации на плоскости параметров $\gamma-k .1-$ периодические колебания, $2-$ квазипериодические колебания, 3 - хаотические колебания. 
Таким образом, в настоящей работе изучена картина нелинейной динамики генератора с запаздывающим отражением от нагрузки, которая в значительной мере определяется многомодовым характером данной системы. При увеличении коэффициента отражения $k$ рождение предельных циклов, соответствующих колебаниям различных мод, происходит либо в результате касательной бифуркации, либо в результате бифуркации Андронова-Хопфа. Дальнейшее увеличение $k$ приводит к возникновению квазипериодических и хаотических колебаний. В областях перекрытия зон генерации различных мод присутствует мультистабильность. Области мультистабильности расширяются с ростом времени задержки, причем в зависимости от параметров в них может наблюдаться сосуществование различных устойчивых режимов (периодических, квазипериодических или хаотических). Обнаружено, что сценарий перехода к хаосу (разрушение трехмерного тора или последовательность удвоений тора) определяется параметром неизохронности $\gamma$. Порог перехода к хаосу увеличивается с уменьшением этого параметра, а при достаточно малых $\gamma$ хаотическая динамика вообще исчезает.

Выявлено влияние величины обратной связи и неизохронности на переходы к хаосу.

Авторы благодарны М.И. Петелину и Ю.В. Новожиловой за стимулирующие дискуссии.

\section{Список литературы}

[1] Erneux T., Javaloyes J., Wolfrum M., Yanchuk S. // Chaos. 2017. V. 27. P. 114201.

[2] Time delay systems: theory, numerics, applications, and experiments / Eds T. Insperger, T. Ersal, G. Orosz. Cham, Switzerland: Springer, 2017. $362 \mathrm{p}$.

[3] Глявин М.Ю., Денисов Г.Г., Кулыгин М.Л., Новожилова Ю.В. // Письма в ЖТФ. 2015. Т. 41. В. 13. С. 25-32.

[4] Kharchev N., Cappa A., Malakhov D., Martinez J., Konchekov E., Tolkachev A., Borzosekov V., Sarksyan K., Petelin M. // J. Infrared Millimeter Terahertz Waves. 2015. V. 36. N 12. P. $1145-1156$.

[5] Khutoryan E.M., Idehara T., Melnikova M.M., Ryskin N.M., Dumbrajs O. // J. Infrared Millimeter Terahertz Waves. 2017. V. 38. N 7. P. $824-837$.

[6] Новожсилова Ю.В., Рыскин Н.М., Усачева С.А. // ЖТФ. 2011. T. 81. B. 9. C. 16-22.

[7] Shigaev A.M., Dmitriev B.S., Zharkov Y.D., Ryskin N.M. // IEEE Trans. Electron Dev. 2005. V. 51. N 5. P. 790-797.

[8] Розенталь Р.М., Гинзбург Н.С., Зайщев Н.И., Иляков Е.В., Кулагин И.С. // ЖТФ. 2006. Т. 76. В. 1. С. 82-85.

[9] Landa P.S. Nonlinear oscillations and waves in dynamical systems. Dordrecht: Springer, 2013. 500 p.

[10] Engelborghs K., Luzyanina T., Roose D. // ACM Trans. Math. Soft. 2002. V. 28. N 1. P. 1-21. 